\title{
Some Applications of Mathematical Programming Techniques in Optimal Power Dispatch
}

\author{
J. Guddat, W. Römisch, and R. Schultz, Berlin \\ Dedicated to the memory of Hansjörg Wacker
}

Received February 13, 1992; revised August 21, 1992

\begin{abstract}
Zusammenfassung
Some Applications of Mathematical Programming Techniques in Optimal Power Dispatch. Some models for the economic dispatch of electric power are introduced and treated by mathematical programming techniques. In particular, our presentation includes (i) a short-term model for the optimal dispatch of thermal units, which is solved by a specific path following method, (ii) a daily model for a generation system consisting of thermal units, pumped storage plants and an energy contract, which can be solved by standard convex quadratic programming algorithms, and (iii) two stochastic programming models for the optimal daily dispatch, which depend on the (unknown) probability distribution of the electric power demand. One of the latter models can be solved efficiently by combining nonparametric estimation procedures and convex programming methods.
\end{abstract}

AMS Subject Classifications: $90 \mathrm{C} 15,90 \mathrm{C} 25$

Key words: Nonlinear programming, stochastic programming, power dispatch.

Einige Anwendungen der mathematischen Optimierung bei der optimalen Lastverteilung. In der Arbeit werden einige Modelle zur optimalen Lastverteilung von Elektroenergie diskutiert und mit Hilfe von Optimierungsmethoden behandelt. Insbesondere gehen wir ein auf (i) ein Modell der MomentanOptimierung der Lastverteilung von Wärmerkraftwerden, das mit einer speziellen parametrischen Optimierungsmethode gelöst wird, (ii) ein Tagesmodell für ein Erzeugersystem bestehend aus Wärmekraftwerken, Pumpspeicherwerken und einem Energievertrag, welches mit Standardmethoden der quadratischen konvexen Optimierung behandelt wird, und (iii) zwei stochastische Optimierungsmodelle für die Optimierung im Tagesbereich, welche von der (unbekannten) Wahrscheinlichkeitsverteilung des Elektroenergiebedarfs abhängen. Eines dieser Modelle kann durch die Kombination nichtparametrischer Schätzmethoden mit Verfahren der konvexen Optimierung effizient numerisch behandelt werden.

\section{Introduction}

The models described in this paper have been developed for the energy situation in East Germany and coordinated with engineers of an electricity-supply enterprise. Our highly esteemed colleague Hansjörg Wacker initiated and pursued this application of optimization techniques. The long and fruitful cooperation with him and his group (in particular in the field of path following methods in parametric optimization, which is of importance in this paper, too) has introduced us to their 
work on energy optimization (see e.g. [1], [15]) and has allowed us to benefit from the experience gathered in Austria.

Our models follow some hierarchy and are designed to support decisions of a dispatcher when running a power generation system comprising thermal units, pumped storage plants and import/export contracts. It is assumed that a proper identification of generating units (unit commitment) is carried out before. Transmission losses in the electric network are modelled by an adjusted portion of demand. Our presentation includes a short-term model and three models with a time horizon of up to a few days: one model where the data are considered to be deterministic, and two further models where the electric power demand enters as a random quantity.

\section{Short-Term Dispatch of Thermal Units}

The task is to allocate amounts of electric power to thermal units of generation such that the total generation costs are minimal, while an electric power demand is met and restrictions for the power output of each unit are satisfied. It is quite usual [2] to model the fuel costs of each unit as a strictly convex quadratic function of the power. Of course, generation costs of different units do not influence each other, and we end up with minimizing some function

$$
y^{T} H y+h^{T} y
$$

subject to

$$
\sum_{i=1}^{K} y^{i}=t \quad \text { and } \quad \underline{a}^{i} \leq y^{i} \leq \bar{a}^{i} \quad(i=1, \ldots, K) .
$$

Here, $K$ denotes the number of generating units and the unknown levels of production are $y^{1}, \ldots, y^{K}$. The cost coefficients are given by the entries in $h \in \mathbb{R}^{K}$ and in the positive definite diagonal $K \times K$-matrix $H$.

Accordingly, $a$ and $\bar{a} \in \mathbb{R}^{K}$ contain the bounds for the power output. The electric power demand is denoted by $t \in \mathbb{R}$.

The specific feature of (2.1), (2.2) is that the power demand $t$ is considered as a real parameter varying between

$$
a_{\min }:=\sum_{i=1}^{K} \underline{a}^{i} \quad \text { and } \quad a_{\max }:=\sum_{i=1}^{K} \bar{a}^{i},
$$

i.e. between the minimum and maximum of the total output of the $K$ units. In this way one arrives at a one-parametric convex quadratic minimization problem. The latter is solved by a very specific path following method (continuation method), whose details are developed in [10]. Since results for more general parametric programs imply that the optimal solutions $y(t)$ of (2.1), (2.2) (for $a_{\min } \leq t \leq a_{\max }$ ) form a continuous, piecewise affine trajectory, it remains to compute those points in $\left[a_{\min }, a_{\max }\right]$ where kinks occur and to find the optimal solutions at these points. Anal- 
yzing the Kuhn-Tucker necessary (and here also sufficient) optimality conditions this can be done quite easily and, in contrast to more general path following methods (cf. [5], [8]), one ends up with an explicit representation of $y(t)$ for $t \in\left[a_{\min }, a_{\max }\right]$. Hence, in the specific setting adopted here, power dispatch reduces to simply inserting the actual demand into the representation of $y(t)$, which, in turn, embodies a very efficient procedure for following a demand curve with a fixed configuration of thermal units. Of course, the method also performs well for an optimal allocation of power for one fixed demand. However, for the latter case there is also a more efficient procedure which is described in [2], [3].

\section{Optimal Daily Dispatch with Deterministic Data}

We extend the model from Section 2 to a generation system consisting of thermal units, pumped storage plants and an energy contract. The generation process is considered for a time horizon up to one day (or one week) with a discretization into hourly or half-hourly intervals.

Let $K$ and $M$ denote the number of thermal units and pumped storage plants. Let $N$ be the number of subintervals in the time discretization. The unknown levels of production in the thermal units and the pumped storage plants are $y_{r}^{i}(i=1, \ldots, K$; $r=1, \ldots, N)$ and $s_{r}^{j}(j=1, \ldots, M ; r=1, \ldots, N$; generation mode $), w_{r}^{j}(j=1, \ldots, M$; $r=1, \ldots, N$; pumping mode). By $z_{r}(r=1, \ldots, N)$ we denote the unknown amounts of energy purchased or sold according to the contract.

The total generation costs comprise the fuel costs of the thermal units (which are given as in Section 2) and the costs (resp. takings) according to the energy contract (which are a linear function of the power). For the pumped storage plants we assume that there occur no generation costs, and hence we have to minimize

$$
y^{T} H y+h^{T} y+g^{T} z
$$

where $y \in \mathbb{R}^{K N}, z \in \mathbb{R}^{N}, H$ is a positve definite diagonal $K N \times K N$-matrix, $h \in \mathbb{R}^{K N}$, $g \in \mathbb{R}^{N}$.

In the constraints we have again an equilibrium between total generation and demand

$$
\sum_{i=1}^{K} y_{r}^{i}+\sum_{j=1}^{M}\left(s_{r}^{j}-w_{r}^{j}\right)+z_{r}=d_{r} \quad \text { for all } \quad r=1, \ldots, N,
$$

and, furthermore, we have conditions characterizing the operation of the different plants

$$
\begin{gathered}
\underline{a}_{1} \leq y \leq \bar{a}_{1}, \quad 0 \leq s \leq \bar{a}_{2}, \quad 0 \leq w \leq \bar{a}_{3}, \quad \underline{a}_{4} \leq z \leq \bar{a}_{4}, \\
S_{j}^{00}-S_{j}^{0} \leq \sum_{r=1}^{\tau}\left(s_{r}^{j}-\eta_{j} w_{r}^{j}\right) \leq S_{j}^{00}, \quad j=1, \ldots, M, \quad \tau=1, \ldots, N, \\
\sum_{r=1}^{N}\left(s_{r}^{j}-\eta_{j} w_{r}^{j}\right)=b_{1 j}, \quad j=1, \ldots, M, \quad \sum_{r=1}^{N} z_{r}=b_{2} .
\end{gathered}
$$


While (3.3) models restrictions for the power output, the inequalities (3.4) reflect the balance between generation and pumping (measured in energy) in the pumped storage plants. $S_{j}^{00}$ and $S_{j}^{0}$ denote, the initial and maximal stocks in energy respectively, in the upper dam. For each pumped storage plant, we assume that the maximal stock in water of the upper dam equals that of the lower dam and that no additional inflow or outflow occurs. Then we put the pumping efficiency, denoted by $\eta_{j}$, as the quotient of the energy gained when letting the full content of the upper dam go down and the energy needed when pumping the full content of the lower dam upward. The equations (3.5) are balances over the whole time period for the pumped storage plants and the energy contract, respectively. Further linear constraints can be appended, for instance to model fuel quotas for the thermal units or intermediate water levels in the dams of the pumped storage plants. Including pumped storage plants, of course, turns the model from a time-separable one into one with constraints linking different time periods. Also, pumped storage plants do not contribute to an optimal solution if the demand curve is traceable by the thermal units alone. However, for the electricity company mentioned at the beginning it was the typical situation that the thermal units alone did not suffice to follow the demand curve. Pumped storage plants were used to manage peaks (and valleys) of the demand. Altogether we have the following convex quadratic program

$$
\min \{g(x): x \in C, A x=d\},
$$

where $x=(y, s, w, z) \in \mathbb{R}^{m}, m:=N(K+2 M+1), g(x)$ is defined by $(3.1), C \subset \mathbb{R}^{m}$ is the bounded convex polyhedron given by (3.3)-(3.5) and the equation $A x=d$ describes (3.2).

(3.6) is solved by standard packages. It has the interesting feature that it circumvents Boolean variables to avoid simultaneous generation and pumping in the pumped storage plants. Of course, it is possible to find points satisfying (3.2)-(3.5) for which both $s_{r_{0}}^{j_{0}}$ and $w_{r_{0}}^{j_{0}}$ are non-zero for some $j_{0} \in\{1, \ldots, M\}$ and some $r_{0} \in\{1, \ldots, N\}$. But such points cannot be optimal, since, due to the strict monotonicity of (3.1) with respect to each $y_{r}^{i}(i=1, \ldots, K ; r=1, \ldots, N)$, we obtain a point with a lower objective value when replacing $s_{r_{0}}^{j_{0}}$ by $s_{r_{0}}^{j_{0}}-\eta_{j_{0}} w_{r_{0}}^{j_{0}}$ and $w_{r_{0}}^{j_{0}}$ by zero (in case $\left.s_{r_{0}}^{j_{0}}-\eta_{j_{0}} w_{r_{0}}^{j_{0}} \geq 0\right)$ or $s_{r_{0}}^{j_{0}}$ by zero and $w_{r_{0}}^{j_{0}}$ by $-\frac{1}{\eta_{j_{0}}} s_{r_{0}}^{j_{0}}+w_{r_{0}}^{j_{0}}\left(\right.$ in case $\left.s_{r_{0}}^{j_{0}}-\eta_{j_{0}} w_{r_{0}}^{j_{0}}<0\right)$ and leaving the remaining components fixed. Therefore, the infeasibility of the problem can be detected by the package either when it finds that there is no vector $(y, s, w, z)$ fulfilling (3.2)-(3.5) or when it presents an "optimal solution" that has $s_{r_{0}}^{j o}>0$ and $w_{r_{0}}^{j_{0}}>0$ for some $j_{0}$ and $r_{0}$. The latter indicates that the demand compared to the minimal output of the thermal units is slightly too low. Hence the units must be re-committed.

\section{Optimal Daily Dispatch with Stochastic Data}

When running the model (3.1)-(3.5) in practice, there is the problem that we want to find some optimal generation policy for a prospective time period without exact 
knowledge of the demand vector $d$, which is typically random. Therefore, a load forecasting procedure is usually carried out before entering the power dispatch model. The exact probability distribution of the demand $d$ is hardly available. A naive approach, however, consists in taking the expectation, and then the model from Section 3 works. For the dispatcher this may be unsatisfactory since it does not exclude infeasibilities in the course of the generation period. He might have some emergency programs (which cause further costs) or at least he wants to be sure that the proposed generation policy is feasible "with a high probability". The main objective of the models presented next is to support the dispatcher in solving a "here-and-now" decision problem, namely planning the prospective generation (with only stochastic information on the demand) in such a way that emergency programmes, "in the mean", have to be used rarely or cause minimal costs. The models do not yield emergency decisions. These have to be found almost on-line after realization of the random data (for instance, with the help of the short-term model from Section 2). Taking these wishes into account and trying to exploit from the random data more than only their expectations leads to stochastic programming ([9], [16]; see also [11]). The idea of "feasibility with high probability" leads to probabilistic (or chance) constraints. So let $\mu$ be some probability measure on $\mathbb{R}^{N}$ reflecting the distribution of the demand vector $d \in \mathbb{R}^{N}$ (cf. (3.2)). Claiming that a generation policy $(y, s, w, z)$ fulfils the demand with probability $p \in(0,1)$ then means that

$$
\mu\left(\left\{d \in \mathbb{R}^{N}: \sum_{i=1}^{K} y_{r}^{i}+\sum_{j=1}^{M}\left(s_{r}^{j}-w_{r}^{j}\right)+z_{r} \geq d_{r}, r=1, \ldots, N\right\}\right) \geq p .
$$

If we replace in (3.1)-(3.5) the constraints (3.2) by the inequality (4.1), we loose polyhedrality, since the left-hand side in (4.1) is a non-linear function of $(y, s, w, z)$ in general. A further problem arises since we often have only incomplete information on the measure $\mu$ in practice and, hence, (4.1) should at least be stable in some sense. A corresponding stability theory is developed in [12]. For the optimization problem given by (3.1), (4.1), (3.3)-(3.5) it yields that optimal generation policies and optimal costs behave stable with respect to perturbations of $\mu$ under some quite natural assumptions.

Picking up the idea of emergency programs after the realization of the random data one arrives at stochastic programs with recourse, which we will discuss next. As in [4] and [6] we introduce a penalty cost for the deviation of the scheduled output from the actual demand, for under- and overdispatching, respectively. Adding the expected penalty (or recourse) costs to the (deterministic) cost function $g$ in (3.6) we obtain the following stochastic power dispatch model:

$$
\min \left\{g(x)+\sum_{r=1}^{N} \int_{-\infty}^{\infty} Q_{r}\left(t-\chi_{r}\right) d F_{r}(t): x \in C, A x=\chi\right\},
$$

where $Q_{r}(t):=\left\{\begin{array}{rl}q_{r}^{+} t, & t \geq 0 \\ -q_{r}^{-} t, & t<0\end{array}, q_{r}^{+}\right.$and $q_{r}^{-}$denote the recourse cost coefficients for under- and overdispatching at the $r$-th time interval, respectively, and $F_{r}$ denotes the probability distribution function of $d_{r}(r=1, \ldots, N)$. 
It is well-known that (4.2) is a particular stochastic program with simple recourse (cf. [9]). Under the basic assumption that

$$
q_{r}^{+}+q_{r}^{-} \geq 0 \quad \text { and } \quad \int_{-\infty}^{\infty}|t| d F_{r}(t)<+\infty \quad(r=1, \ldots, N),
$$

(4.2) is a convex (nonlinear) program having linear constraints. The objective function of (4.2) is (twice) continuously differentiable if all distribution functions $F_{r}$ $(r=1, \ldots, N)$ have (continuous) densities (cf. [9]). In [4] it is suggested to choose $F_{r}(r=1, \ldots, n)$ as a (trimmed) normal distribution. However, our tests with the available empirical data have not justified this hypothesis. We finally preferred to use the following smooth nonparametric estimates for $F_{r}$ :

$$
\hat{F}_{r}^{(n)}(t):=\frac{1}{n b_{n}} \sum_{i=1}^{n} \int_{-\infty}^{t} k\left(\frac{x-\xi_{i r}}{b_{n}}\right) d x \quad(t \in \mathbb{R} ; n \in \mathbb{N}),
$$

where $\xi_{1 r}, \ldots, \xi_{n r}$ is an independent sample taken from the distribution $F_{r}$ $(r=1, \ldots, N), k: \mathbb{R} \rightarrow \mathbb{R}$ a nonnegative function having the property $\int_{-\infty}^{\infty} k(x) d x=1$ ("kernel") and $\left(b_{n}\right)$ a sequence of positive numbers tending to zero ("smoothing parameters"). For more information on such kernel-type estimators, in particular on possible choices of $k$ and $\left(b_{n}\right)$ together with asymptotic results as the sample size $n$ tends to infinity, we refer to [6] and the literature cited therein. These asymptotic arguments together with stability results for stochastic programs ([13]) yield a theoretical foundation of the approach to replace $F_{r}$ in (4.2) by its kernel estimate $\hat{F}_{r}^{(n)}(r=1, \ldots, N)$. One result in this direction will be described next. Let $\psi$ denote the set of solutions to (4.2) and $\hat{\psi}_{n}$ the corresponding solution set if $F_{r}$ is replaced by $\hat{F}_{r}^{(n)}(r=1, \ldots, N)$.

Proposition: Let $q_{r}^{+}+q_{r}^{-}>0$ for all $r=1, \ldots, N$. Assume, in addition, that the functions $F_{r}, r=1, \ldots, N$, are twice differentiable such that $F_{r}^{\prime \prime}$ is bounded on $\mathbb{R}$, and that there exist an open neighbourhood $U$ of $A \psi$ and a constant $\varepsilon>0$ such that

$$
\prod_{r=1}^{N} F_{r}^{\prime}\left(t_{r}\right) \geq \varepsilon, \quad \text { for all }\left(t_{1}, \ldots, t_{N}\right) \in U
$$

Furthermore, let all distributions $F_{r}(r=1, \ldots, N)$ have compact support and let $k$ be a class 2 kernel, i.e. $\int_{-\infty}^{\infty} x k(x) d x=0$ and $\int_{-\infty}^{\infty} x^{2} k(x) d x<+\infty$. Then we have the following rate of mean convergence of optimal sets

$$
E\left[d_{H}\left(\psi, \hat{\psi}_{n}\right)\right]=O\left(n^{-1 / 4}\right),
$$

whenever $\left(b_{n}\right)$ is chosen such that lim $\sup _{n \rightarrow \infty} b_{n}^{2} n^{1 / 2}<+\infty$. Here $d_{H}$ denotes the Hausdorff distance on bounded subsets of $\mathbb{R}^{m}$.

This result is proved in [6] (combine Theorem 3.5 and Proposition 4.2). Its proof relies essentially on a general quantitative stability result for stochastic programs with complete recourse (Theorem 2.7 in [13]). More recent investigations [14] even lead to the improved (and best possible) rate of convergence

$$
E\left[d_{H}\left(\psi, \hat{\psi}_{n}\right)\right]=O\left(n^{-1 / 2}\right) .
$$


Justified by the above theory, our numerical treatment of the model (4.2) begins with replacing $F_{r}$ by $\hat{F}_{r}^{(n)}$ taken from $(4.3)(r=1, \ldots, N)$. This leads to the foilowing convex program having smooth data and polyhedral constraints:

$$
\min \{g(x)+\hat{Q}(\chi): x \in C, A x=\chi\} .
$$

Explicit formulas for $\hat{Q}$ and its gradient in terms of the recourse cost coefficients, the samples, the smoothing parameters and of the functions $\mathscr{K}_{1}(t):=\int_{-\infty}^{t} k(x) d x$, $\mathscr{K}_{2}(t):=\int_{-\infty}^{t} x k(x) d x(t \in \mathbb{R})$ can be derived immediately (Section 5 of [6]). Since, for most kernels $k$, the real functions $\mathscr{K}_{1}$ and $\mathscr{K}_{2}$ can be calculated explicitly, no numerical integration has to be performed when evaluating $\hat{Q}$ and its gradient. The second step in the treatment of (4.2) is the solution of (4.4) by standard convex programming algorithms. A program system STOCHOPT according to this approach has been implemented on a PC 386 by Mrs. N. Gröwe. Numerical results and conclusions of a series of test runs for a stochastic power dispatch model of East Germany $(K:=24, M:=5, N:=24$ and, hence, $m=840)$ are reported in [7]. Two observations deserve special attention: (1) The running times on a PC 386 did not exceed 5 minutes and had the same order of magnitude as for the corresponding deterministic model (3.6). (2) The optimal costs of the stochastic model are lower than those for the deterministic one even if a reserve level of (only) $3 \%$ of the demand is adjusted (in (3.6)).

\section{Acknowledgement}

The authors wish to thank an anonymous referee for constructive criticism.

\section{References}

[1] Bauer, W., Gfrerer, H., Lindner, E., Schwarz, A., Wacker, H.: Optimization of the Gosau hydroenergy powerplant system. Mathematical Engineering in Industry 1, 169-190 (1987).

[2] van den Bosch, P. P. J.: Optimal static dispatch with linear, quadratic and nonlinear functions of the fuel costs. IEEE Transactions on Power Apparatus and Systems PAS-104, 3402-3408 (1985).

[3] van den Bosch, P. P. J., Lootsma, F. A.: Scheduling of power generation via large-scale nonlinear optimization. Journal of Optimization Theory and Applications 55, 313-326 (1987).

[4] Bunn, D. W., Paschentis, S. N.: Development of a stochastic model for the economic dispatch of electric power. European Journal of Operational Research 27, 179-191 (1986).

[5] Gfrerer, H., Guddat, J., Wacker, H.: A globally convergent algorithm based on imbedding and parametric optimization. Computing 30, 225-252 (1983).

[6] Gröwe, N., Römisch, W.: A stochastic programming model for optimal power dispatch: Stability and numerical treatment. In: Marti, K. (ed.) Stochastic optimization. Berlin: Springer 1992 pp. 111-139 (Lecture Notes in Economics and Mathematical Systems, Vol 379).

[7] Gröwe, N., Römisch, W.: Numerical treatment of a stochastic programming model for optimal power dispatch. Proceedings of the Sixth Annual Conference of ECMI (Limerick, Ireland 1991; Hodnett, F. ed.), Dordrecht: Kluwer (to appear).

[8] Guddat, J., Guerra Vasquez, F., Jongen, H. Th.: Parametric optimization: singularities, pathfollowing and jumps. Chichester: Wiley 1990.

[9] Kall, P.: Stochastic linear programming. Berlin: Springer 1976.

[10] Kleinmann, P., Schultz, R.: A simple procedure for optimal load dispatch using parametric programming. Zeitschrift für Operations Research 34, 219-229 (1990).

[11] Prékopa, A.: Recent results in optimization of electro-energetic systems. In: Wacker, $H$. (ed.) Applied optimization techniques in energy problems. Stuttgart: Teubner 1985, pp. 354-383. 
[12] Römisch, W., Schultz, R.: Distribution sensitivity for certain classes of chance-constrained models with application to power dispatch. Journal of Optimization Theory and Applications 71, 569-588 (1991).

[13] Römisch, W., Schultz, R.: Stability analysis for stochastic programs. Annals of Operations Research $30,241-266(1991)$.

[14] Römisch, W., Schultz, R.: Lipschitz stability for stochastic programs with complete recourse, manuscript, Humboldt-Universität Berlin, Fachbereich Mathematik, 1992.

[15] Wacker, H. (ed.): Applied optimization techniques in energy problems. Stuttgart: Teubner 1985.

[16] Wets, R. J.-B.: Stochastic programming. In: Nemhauser, G. L., Rinnoy Kan, A. H. G., Todd, M. J. (eds.) Handbooks in operations research and management science, Vol. 1, Optimization. Amsterdam: North-Holland 1989, pp. 573-629.

\author{
J. Guddat \\ W. Römisch \\ R. Schultz \\ Humboldt-Universität Berlin \\ Fachbereich Mathematik \\ D-O-1086 Berlin \\ Federal Republic of Germany
}

